\title{
The International Salmon Data Laboratory (ISDL)
}

\author{
Scott A. Akenhead \\ Pacific Biological Station, 3190 Hammond Bay Road, Nanaimo, BC, V9T 6N7, Canada
}

Keywords: Pacific salmon, salmon, data processing, International Year of the Salmon, analysis, database

Given that salmon ecologists wish to improve the resilience of salmon to unmitigated anthropic climate change, a paradigm shift is required: from monitoring, passive conservation, optimal harvesting, and enhancement (spawning channels and hatcheries) to predicting how salmon will respond to habitat and ecosystem changes that exceed the range of historical observations. To be useful, that new prediction ability must be translated into effective actions. The mobilization of this knowledge will lead to salmon fisheries and habitat managers being better informed and able to react quickly to unexpected events. The International Year of the Salmon (IYS) has presented this as a challenge to the world. The International Salmon Data Laboratory (ISDL) is a response to that challenge.

The first ISDL workshop was held in Vancouver, Canada, on 25 January 2019 (NPAFC 2019b) with the following general objective: After salmon ecologists say what they need in terms of data processing, ISDL describes how that can be delivered with modern technology (Fig. 1). The following summarizes the ISDL workshop's progress toward this objective.

Fig. 1. A poster presented the assembly, standardization, and integration of many salmon datasets - a prerequisite to efficient, broader, and deeper analysesas the metaphorical re-assembly of a salmon from many kinds of salmon sushi (diverse datasets) and the subsequent production of a new generation of salmon (analyses, data products).
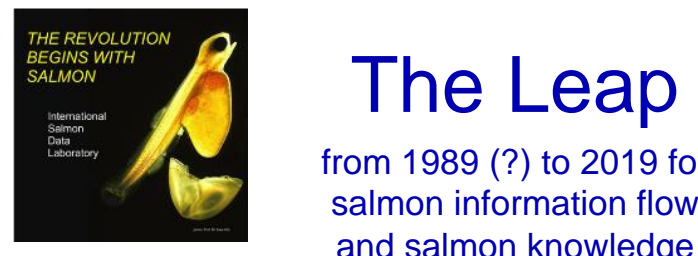

from 1989 (?) to 2019 for salmon information flow and salmon knowledge

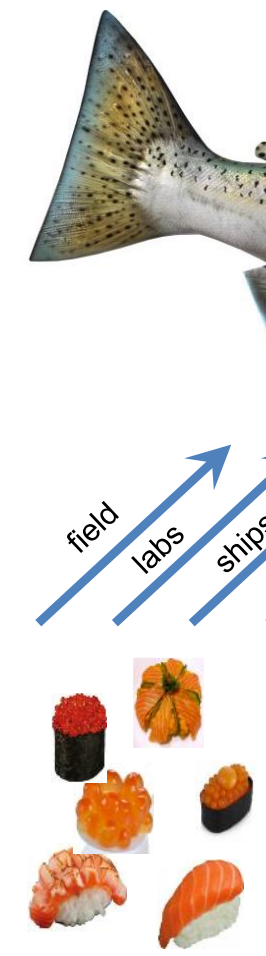

metaphorical sushi: salmon datasets are myriad, disparate, disorganized,.
Contact: Scott Akenhead scott@s4s.com and John Song jsong222@gmail.com

INTERNATIONAL YEAR ${ }_{\text {THE }}^{\text {OF }}$ SALMON

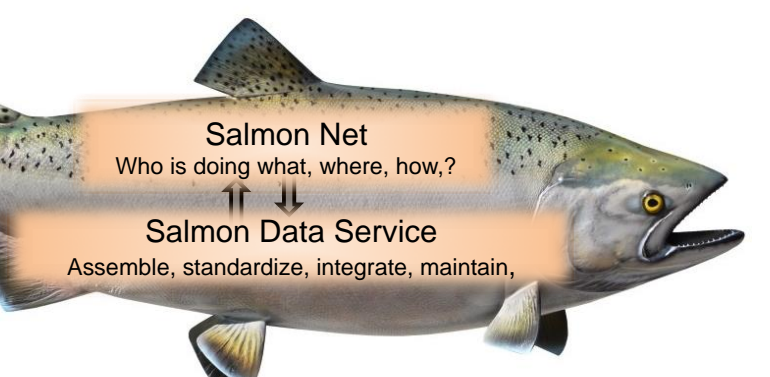

doi:10.23849/npafctr15/203.204. 
Prediction ability requires mechanistic models as opposed to correlations. Developing and applying those models requires that we:

(a) Shift to a new perspective on what drives salmon population dynamics. This involves comparing the success and failure of many salmon populations across multiple species each with several life-history strategies, across the northern hemisphere, in many situations. This was a goal of the immediately preceding IYS Salmon Status and Trends Workshop (NPAFC 2019a).

(b) Assemble and integrate an unprecedented breadth and depth of information about salmon and the habitats they encounter. The ISDL workshop examined a specific recent technology, the neo4j graph database, with associated software libraries and developers' tools, as the basis for a breakthrough in data mobilization. This capacity was explained by Jeff Morris (San Mateo, CA) noting that Neo4j Inc is supporting ISDL via their Graphs for Good program. A practical elucidation of the flexibility of schema for a graph database compared to a relational database, and a cogent demo wherein large salmon datasets were uploaded and visualized via neo4 $\mathrm{j}$ was delivered by John Song (Los Angeles, CA).

(c) Modernize salmon data processing from field collections, to integration and analysis. Immediate mobilization of all types and formats of field data with the GeoOptix platform was described by Matt Denniston and Keith Steele (Portland, Oregon). Scott Akenhead pointed to the development of technologies that are capable of modernizing estimates of spawner abundance (resistivity counters) and estimates of age-1 smolt survival (tiny acoustic tags), just two examples of many possible. Bruce Patten (Nanaimo, BC) reviewed how Fisheries and Oceans (DFO) Pacific Region manages hundreds of data sources, many databases, and many data customers, highlighted by a SWOT analysis (strengths, weaknesses, opportunities, threats) of the migration from 1990's technology and practices to new tools, systems, and practices.

(d) Mobilize data for improved decision support. Sue Grant (Vancouver, BC) reviewed this requirement with reference to DFO's State Of Salmon (SOS) program and to DFO's support for the Pacific Salmon Explorer that provides interactive data visualization (sponsored by Pacific Salmon Foundation and DFO).

(e) Transfer knowledge from scientists to policy makers and to salmon fisheries and habitat managers. In an illuminating moment, Kelly Chapman (Powell River, BC) changed the thinking of everyone at the workshop by describing how this requires extensive personal engagement, in sharp contrast to the implicit assumption that "products" were sufficient in all preceding presentations.

Several ecologists offered to carry the ISDL ideas to international workshops in May and September of this year-an encouraging result that emphasized the potential value of the ISDL initiative. The suggestions for projects from this workshop and from preceding IYS workshops were combined as a strategic plan for ISDL: goals, quantifiable objectives, projects, experiments. A suite of ideas for "experiments" for this "data laboratory" were discussed while noting that ISDL plans require resourcing:

- The ecologists asked for immediate action to standardize (via glossary of parts and methods) and integrate the datasets that will be tabled via IYS Salmon Status and Trends.

- Experiments with GeoOptix (GeoOptix.com) for data capture directly to cloud servers, circumventing many existing problems (completeness, quality control, timeliness, and multiple formats).

- Demonstrations that the neo4j technologies will deliver the required breakthrough in data integration.

- Automation of data updates, for the maintenance of a database assembled from many (changing) sources,

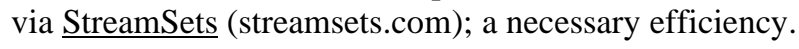

- Experiments with analysis workflows via WorkSpace (https://research.csiro.au/workspace/) to deliver results from the integrated database. Reducing the repetitive data assembly, standardization, and integration that precedes every analysis would be a welcomed benefit.

\section{REFERENCES}

NPAFC. 2019a. Report of the Proceedings for the IYS Workshop: International Year of the Salmon Workshop on Salmon Status and Trends, Vancouver, BC, Canada, January 23-24, 2019. Edited by J.R. Irvine, K. Chapman, and J. Park. N. Pac. Anadr. Fish Comm. Tech. Rep. 13. (Available at https://npafc.org)

NPAFC. 2019b. Report of the Proceedings for the IYS Workshop: First International Year of the Salmon Data Laboratory (ISDL) Workshop, Vancouver, BC, Canada, January 25, 2019. Edited by S. Akenhead, N. Bendriem, and J. Park. N. Pac. Anadr. Fish Comm. Tech. Rep. 14. (Available at https://npafc.org) 